\title{
Towards Engineering Transparency as a Requirement in Socio-technical Systems
}

\author{
Mahmood Hosseini, Alimohammad Shahri, Keith Phalp, Raian Ali \\ Faculty of Science and Technology \\ Bournemouth University, United Kingdom \\ \{mhosseini, ashahri, kphalp, rali\}@bournemouth.ac.uk
}

\begin{abstract}
The improvement and success of socio-technical systems depend on the joint optimisation of both the social and the technical parts. Improving the social part of a socio-technical system is a meticulous task, as social requirements are diverse and dynamic, and they usually evolve with time and context. Information transparency (henceforth, transparency) is one of the social requirements that can affect the overall attitude of the stakeholders present within a socio-technical system, and influence their other social requirements such as privacy, trust, collaboration and non-bias. In this paper, we advocate the need to engineer transparency as a first class requirement, propose a baseline model for transparency and show how this model can be a starting point for the analysis of transparency requirements of different stakeholders. We showcase our on-going research in the modelling and analysis of transparency as a requirement, discuss some of the challenges of transparency requirements elicitation, and present our future work.
\end{abstract}

Index Terms - transparency requirements, transparency modelling, transparency analysis, socio-technical systems

\section{INTRODUCTION}

Socio-technical systems (STSs) emphasise the interaction that exists amongst social actors and also between social actors and technical actors in an organisational settings [1]. Mainstream modelling languages in requirements engineering, such as goal modelling [2] and business process modelling [3], reflect such a view of STSs. One part of this sociotechnical interaction is the transfer of information from one social entity to another. The information transfer amongst various social entities brings several topics into question, such as copyright, privacy, security, trust and transparency. Some of these concepts have been studied extensively both from the social viewpoint and from the requirements engineering viewpoint. For example, the notion of privacy is one of the classical concepts in social sciences research, e.g., in [4], and from the engineering perspective, e.g., in an attempt to model privacy requirements in [5].

One of the concepts that is under-researched from the engineering perspective, in comparison to other similarly important concepts, is the concept of transparency. It is generally considered that providing transparency has desirable effects, while the lack of transparency raises doubts on the ethical and professional practice. For example, in the context of politics and governance, it is illustrated that "more transparent governments govern better" [6]. However, we will illustrate in this paper that the right level of transparency should be achieved if joint optimisation, which is the goal of STSs for the emergence of productivity and well-being [7], is to be accomplished; otherwise, providing transparency may lead to adverse effects. For example, it was studied and shown in [8] and [9] that transparency may have significant unintended consequences in medical care. It was found that when 'media spectacles' and transparent regulation are combined, it can cause 'spectacular transparency' which has some perverse effects on doctors' practice and increased defensive behaviour in doctors and their staff. Furthermore, in another four-year organisational study, [10] discovered that in the context of a clinical risk management, when intersubjective relations are solely regulated by transparent self-revelation to authorities, this transparency can undermine ethical behaviour and lead to organisational crisis and even collapse.

These studies clearly illustrate that transparency, when applied in an ad hoc manner, can lead to serious issues in STSs. Therefore, it is important to study transparency and its emergent side-effects. We advocate that transparency can be seen either as a special kind of requirement or as a metarequirement, i.e., a requirement of a social entity to know how their requirements are being fulfilled by others. As a requirement, software engineers have to deal with transparency during the requirements engineering process and requirements definition [11].

Let us take an example of a requirements engineering department as an STS. A requirements engineer's job includes the elicitation of a set of requirements from various stakeholders of a system and the prioritisation and specification of these requirements, with the aim of designing a new system which meets the expectations of its stakeholders. In such a process, requirements engineers might need to make a stakeholder's requirements visible and transparent to another stakeholder. Such transparency may discourage certain stakeholders to voice their requirements. This means that the process of requirements elicitation might be incomplete, and the produced or evolved software based on it will probably be flawed, inefficient or inappropriate in part or for some stakeholders. All these problems can arise when requirements engineers do not consider transparency requirements of their stakeholders during the elicitation process.

One issue to bear in mind is that calling for less transparency does not necessarily mean that it is stemmed only from privacy requirements by the stakeholders, because the transferred information may not necessarily be personal information or 
relate to what a stakeholder would like to hide. Instead, it might be about one stakeholder's preference not to provide such information because they think it may create undesirable effects such as information overload or bias.

In this paper, we take the first steps towards modelling and analysis of transparency in STSs. We advocate that while transparency has its own unique characteristics and peculiarities, there is still a lack of dedicated models, tools or approaches that allow a managed engineering of transparency in STSs which can, amongst other things, estimate the right level of transparency and predict its side effects. We advocate the need for a separate modelling and analysis for transparency, as it is a concept that should be investigated on its own merits. This argument, which is similar to the argument about the modelling and analysis of related social concepts in STSs, e.g., security or privacy [12], is supported by the famous software engineering principles of separation of concerns and modularity. That is, to better manage transparency, we need to consider it as a first class concept and capture its peculiarities and its inter-relations with other requirements models via designated approaches.

The rest of the paper is structured as follows. In Section II, we provide a background to our study and pinpoint its importance. In Section III, we present our baseline model of transparency and elaborate on it. In Section IV, we demonstrate an example of transparency requirements modelling through a mainstream requirements model, the goal model, and illustrate how certain characteristics of transparency necessitate the modelling and analysis of transparency as a separate first class concept. In Section V, we conclude and present the future work.

\section{DEFINITION AND BACKGROUND}

Transparency refers to the possibility of accessing information, intentions or behaviours that are revealed through a process of disclosure [13]. It is usually linked to accountability, efficiency and openness [14]. Transparency is also observed as an important concept as it can support users in the process of decision making [13].

Transparency effects have been studied in several social and technical settings. Transparency has generally been accounted to increase accountability [15], create trust among different stakeholders [16], help democratic decision-making [17], convey honesty and integrity [14], and encourage open decision making [14].

On the other hand, there are only few studies on transparency in requirements engineering. In [18], the authors propose that software transparency should be based on requirements, which in turn will provide a baseline for both upstream and downstream traceability. They also propose a transparency ladder, which shows different steps to achieving transparency. In [19], the authors design a survey to investigate the importance of transparency as it enables stakeholders to identify and understand the information transfer during stakeholders' communications. In [20], the authors propose a framework for capturing the stakeholders' requirements patterns which are related to transparency through argumentation. In [11], the authors define transparency as a non-functional requirement and therefore use a Non-Functional Requirements Framework and Softgoal Independence Graph to represent transparency and several quality attributes related to it. While all these studies have contributed much to our knowledge of transparency, we still lack a model of transparency that can help requirements engineers in the automated reasoning and analysis of transparency requirements of stakeholders. This is our intention in this study of transparency.

\section{Modelling Transparency}

According to [11], there are two types of transparency, information transparency and process transparency. Information transparency refers to the level of information available to stakeholders, while process transparency refers to the level of process clarity. In our study, we refer to these two types as stakeholders' transparency requirements and metarequirements. The reason behind this is that as a metarequirement, transparency becomes a requirement about another requirement. We use the same example that [11] give to make it clear.

In [11], the authors suppose that information exists in a company about safety emission levels for a given artefact. Requiring that this information be available to customers is information transparency. Since the stakeholders ask for such transparency-related information itself, we can call it a transparency requirement. On the other hand, when stakeholders need to know how the artefact is being assembled, they need some information about the process used for artefact assembly, and this is called process transparency. Here, since the stakeholders' real requirement is the artefact, and the transparencyrelated information is a requirement about another requirement, we can call it transparency meta-requirement. In both cases, however, the following concepts should be noted:

- Both in information transparency and process transparency, it is information that is being processed and transferred from one social entity to another. Therefore, while we acknowledge the differences between the two concepts, we advocate that any model of transparency should focus on the flow of information. This vision is also confirmed in [21], when the authors mention that transparency in process models deals with organising and documenting information about processes.

- Whether we look at transparency as a requirement or as a meta-requirement, it is still a requirement and will benefit from engineering approaches. Therefore, while we acknowledge the differences between the two concepts, we refer to both as a requirement.

\section{A. Baseline Model of Transparency}

As described earlier, in our study, transparency is all about information, how it flows and transfers between different entities and how it can be tracked and related to the entity 


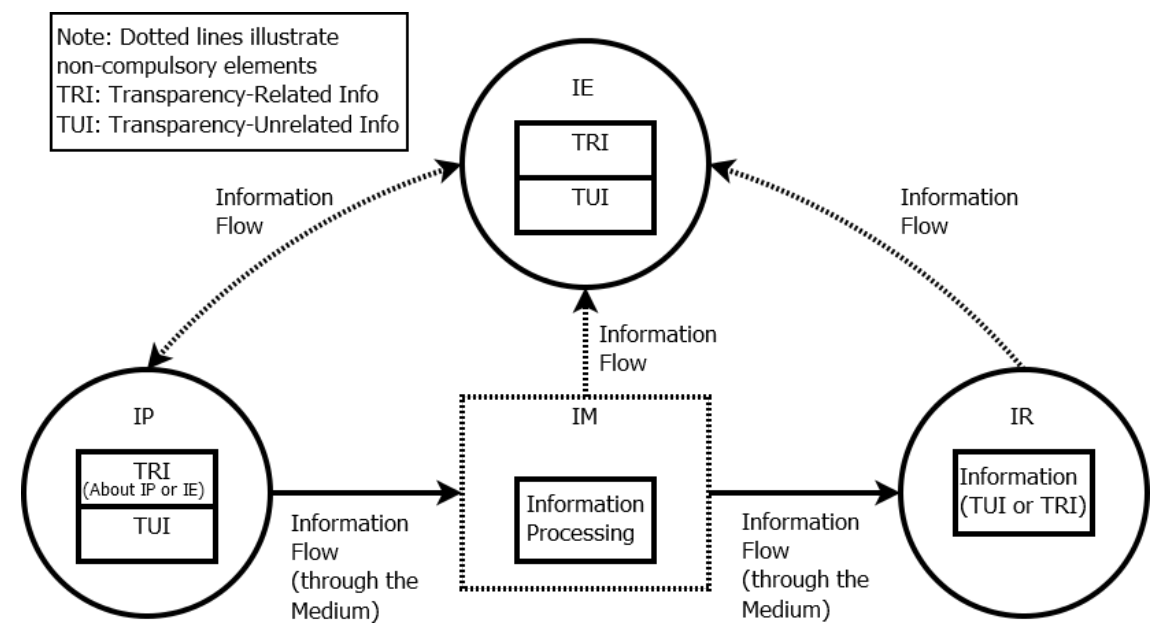

Fig. 1. A representation of information transparency model

providing that information. In our model of transparency, any flow of information can be broken down into four elements:

- Information Provider (IP): the entity that is providing and presenting some information about another entity, or about itself.

- Information Receiver (IR): the entity that is receiving, probably upon request, the information about another entity, or about itself.

- Information Entity (IE): the entity whose information is being transferred. This can sometimes include the IP or the IR, depending on the context.

- Information Medium (IM): the medium through which the information is being channelled and transferred.

There are a few details which should be noted about these four elements of transparency. These are as follows:

1) IP and IE are the same if information provider is giving information about themselves. For example, when someone is describing their own job.

2) IR and IE are the same if information provider is giving information about information receiver. For example, when someone is telling their colleague about that colleague's performance.

3) IP, IR, and IE are the same if information provider is giving information about themselves to themselves. For example, when someone is keeping a private journal about themselves. This can be of importance in the presence of an information medium, as information can be stored and found on it, and this may lead to undesirable transparency.

4) IM might be absent in cases where the information is being transferred without using any medium, e.g., in face-to-face communications.

Therefore, in our model of transparency, only the presence of the information provider and the information receiver is obligatory. With respect to the information that is being transferred, any information can be divided into two subcategories:
- Transparency-Related Information (TRI): This is the information that carries data related to transparency presented by the information provider (IP) about the information entity (IE).

- Transparency-Unrelated Information (TUI): This is the information whose transparency is not important, relevant or in question, presented by the information provider (IP) about the information entity (IE).

Based on the information we have provided so far about transparency, the transparency model is illustrated it in Fig. 1.

\section{B. Levels of Transparency}

Based on the model of transparency we have provided, we can perceive five different levels of transparency:

- Level 1 - No Transparency: If IP only sends TUI information to IR, either through IM or directly, and does not send any TRI information, then we can say that there is no transparency achieved. In this case, only IP knows about TRI.

- Level 2 - Unilateral Transparency: If IP sends TRI information along with TUI information to IM, but IM does not reveal TRI information to IR and only sends TUI information to IR, then we can say a unilateral level of transparency is achieved. In this case, it is still only the IP who knows about TRI, but this information is also stored in IM. It should be noted, however, that certain laws and regulations may oblige IM owners to reveal this information and make it transparent, which means the unilateral level of transparency will no longer be maintained. Furthermore, in our model of transparency, unilateral transparency can never be reached if there is no IM.

- Level 3 - Bilateral Transparency: If IP sends TRI information along with TUI information to the IR, either through IM or directly, then we can say bilateral transparency is achieved. In this case, only IP and IR know about TRI. 
- Level 4 - Trilateral Transparency: If IP sends TRI information along with TUI information to IR, either through IM or directly, and one of IP, IM or IR also sends them to IE, then we can say trilateral transparency is achieved. In this case, IP, IR and IE know about TRI.

- Level 5 - Full Transparency: If IP sends TRI information along with TUI information to IR, either through IM or directly, and probably one of IP, IM or IR also sends the IE, and at the same time they make it accessible to the general public (including IE), then we can say full transparency is achieved. In this case, potentially everyone knows about TRI.

Being in different contexts where transparency requirements arise necessitates different appropriate levels of transparency. Therefore, it is important to investigate which level of transparency is needed in every case study based on the context of that case study.

\section{Transparency Analysis Outcome}

The study of our model of transparency leads to another aspect of transparency, which is to analyse if the right level of transparency has been reached. IP and IR have a required level of transparency, which is the level of transparency they need, and an achieved level of transparency, which is the level of transparency they actually get. As a result, three outcomes are possible:

- Transparency Shortage: Transparency shortage happens when the achieved level of transparency is lower than the required level of transparency. This can lead to conflicts of interest in the level of transparency.

- Transparency Coverage: Transparency coverage happens when the achieved level of transparency is equal to the required level of transparency. This is the optimal solution which helps toward joint optimisation in STSs.

- Transparency Abundance: Transparency abundance happens when the achieved level of transparency is higher than the required level of transparency. This can also lead to conflicts of interest in the level of transparency.

Fig. 2 shows the basic outcome of transparency analysis in an STS, emphasising that transparency requirements are appropriately met only when achieved transparency equals required transparency.

\section{Conflicts of Interest in Transparency Levels}

It is sometimes the case that IP, IR, and possibly IE (i.e., when IE is a social actor) have different transparency requirements. This can lead to conflicts of interest in the level of transparency that each entity requires. We elaborate on this by providing the following examples:

Example 1: Suppose a company is using a cloud service, and for safety and security reasons, they would like to know where their stored files are hosted and how they are encrypted. Therefore they use the cloud service provider platform to get this information from them. The client company may not be willing to use the cloud services if the geographical location of their servers poses a threat to their security. In this example, IP

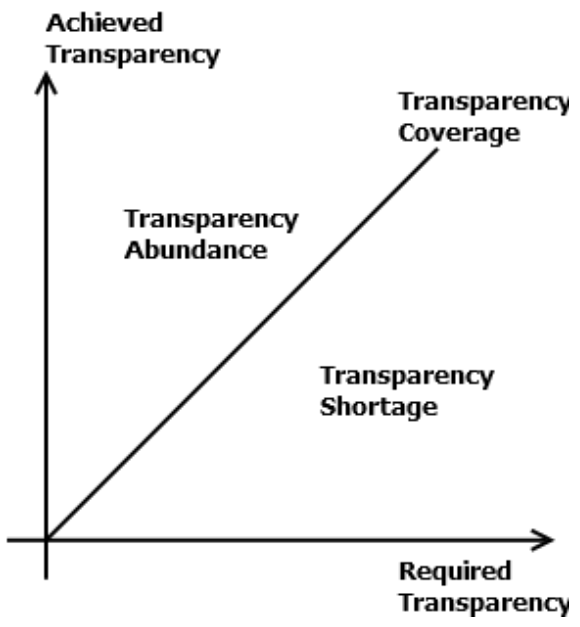

Fig. 2. Basic outcome of transparency analysis

is the cloud service provider, IR is the client company, IE is the server location and IM is the cloud service provider platform. The level of transparency the client company is looking for is bilateral transparency, or probably any level higher than that, i.e., the server location becomes public. If, for example, the cloud service provider refuses to provide such information to the client company, then the level of transparency the cloud service provider is offering is no transparency. On the client company's side, this leads to transparency shortage.

Example 2: Suppose a government agency is investigating a case and needs more information about a suspect. The government agency contacts an email service provider to collect some information about that suspect's correspondence. In this example, IP is the email service provider, IR is the government agency, IE is the suspect and IM is the telephone call. The level of transparency the government agency is looking for is bilateral transparency, and not any level higher or lower than that. If, for example, regulations on the email service provider side oblige it to reveal such exchange of information to the suspect as well, then the level of transparency the email service provider needs is trilateral transparency. On the government agency's side, this leads to transparency abundance.

These examples illustrate the possible conflicts of interest that may arise between different actors and how these conflicts can lead to transparency shortage or abundance. Furthermore, such conflicts can apparently lead to less accountability (as in example 1) or less trust (as in example 2).

\section{Study of Transparency and Its Challenges}

In this section, we present an example to illustrate the importance of and the need for transparency modelling and analysis.

\section{A. Example: Online Transcription Service}

Suppose a researcher is looking for an online transcription service for transcribing some audio files to text, as illustrated via a goal model in Fig. 3. We use goal modelling [22], [23] as it helps to illustrate the rationale of each actor, including 


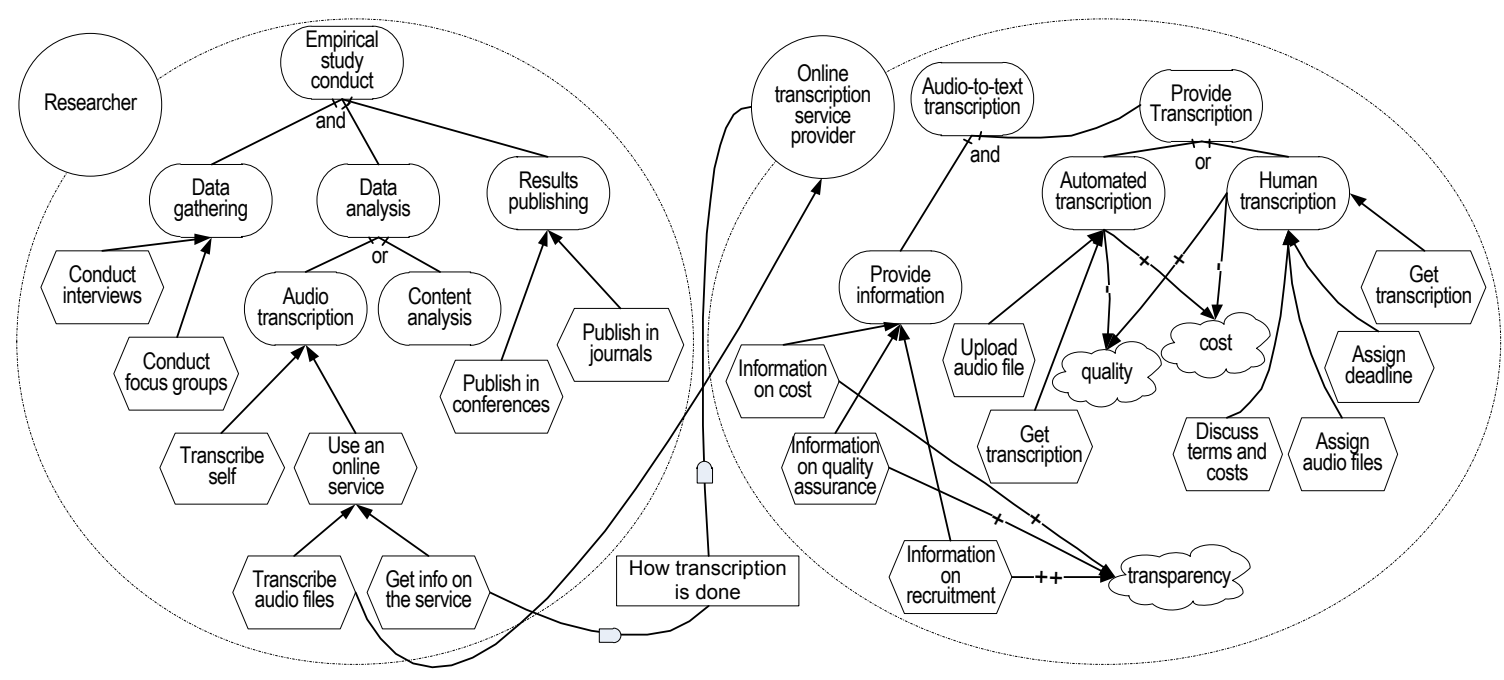

Fig. 3. A goal model demonstration for transparency requirements

the goals they have, the way they achieve these goals and their dependencies on each other in a STS. Such a model could also be a baseline for our transparency model in the future work as transparency is mainly about sharing knowledge with other inter-dependent social entities in an STS. In this example, and based on our baseline model of transparency, IP is the online transcription service provider, IR is the researcher, IE is the information about transcription costs, quality, etc., and IM can be any medium through which the two actors communicate.

It is shown in Fig. 3 that providing some information about the online transcription service, such as how much different services may cost, how different services influence the quality of the transcription, and how people are recruited to perform the transcription, can affect transparency in a positive way, i.e., increase the level of transparency. However, increasing transparency level, as explained before, does not necessarily mean meeting transparency requirements of the involved actors. As a result, while this model provides some information about transparency and how to increase it, we would still want to address several fine-grained transparency concerns which necessitate additional modelling constructs. Such concerns include:

- The ability to inform transparency levels: The proposed model of transparency should utilise designated constructs to inform requirements engineers what level of transparency is required and what level of transparency is achieved. This means that the model should facilitate constructs so that every actor, upon requesting information, can clearly state what level of transparency they require, and upon providing information, what level of transparency they can achieve.

- The ability to compare transparency levels: This model of transparency will then be able to help requirements engineers to compare and analyse whether the achieved level of transparency is the required level as well.
- The ability to detect possible conflicts: Requirements engineers will then be able to identify any conflicts of interest that may arise as a result of the difference between the achieved level of transparency and the required level of transparency. This, in turn, will help them to try to resolve the issue by communicating with the actors, informing them of the issue and reviewing their policies and regulations when applicable.

- The ability to support decision-making: The proposed model of transparency should also be able to illustrate how transparency requirements of different actors and possible conflicts of interest on transparency requirements may influence and support different actors' decisions to choose amongst alternatives to reach a goal. This enables requirements engineers to propose alternatives to different actors to reach the same goal while adhering to their transparency requirements.

- The ability to analyse side effects: Requirements engineers will use the proposed model of transparency and the information it provides to analyse the effect of the achieved transparency on other softgoals, such as trust, accountability, etc. in different actors.

These concerns show the necessity for designated constructs to model transparency as a requirement that can capture and address them.

\section{B. Challenges Related to Transparency}

To aid the engineering of transparency requirements, we still need to handle basic challenges not related to the modelling and analysis but to the elicitation of such requirements. They are as follows:

- Transparency requirements of actors are themselves subject to other meta-transparency requirements. When an actor states their transparency requirements of other actors, they may not necessarily agree or prefer that others are aware of those requirements. This creates a paradox 
that may be resolved by some sort of obfuscation techniques and translucence, both emerging research topics.

- Transparency requirements are typically hard to specify, as such specification requires stakeholders to state what they usually do and believe which they may not necessarily be able to state. The challenge on tacit knowledge in requirements [24] is maximised here. We may rely on a feedback loop to keep the transparency requirements updated by allowing online and runtime requirements elicitation [25] from the users in the real context.

- Transparency requires not only the typical stakeholders, e.g., the different roles in an organisation, but also expertise in ergonomics and human factors in computing. Such a multi-disciplinary topic also calls for a multidisciplinary requirements engineering team. We still need to think what sort of expertise such a team should have and how to manage the viewpoints within the team [26].

\section{CONCLUSION}

In this paper, we show how requirements engineers can benefit from a transparency model which captures the transparency requirements of different social actors, and aids in the analysis of those requirements and their possible side effects. We discuss how inefficient handling of transparency requirements may lead to possible adverse effects, such as reducing the level of trust or decreasing accountability, and how these adverse effects may arise as a result of transparency shortage or transparency abundance. Consequently, we propose that modelling and analysis of transparency as a requirement is a step forward to a better engineering of this concept in STSs. Our baseline model of transparency seeks to conceptualise the flow of information amongst different actors, and facilitates the establishment of an automated analysis that aids requirements engineers and different stakeholders to understanding the different levels of transparency, how conflicts of interest may arise as a result of unmatched transparency requirements and how these conflicts might be resolved.

In our future research, we aim to develop this baseline model further to be able to capture transparency-related data in conjunction with a mainstream STS requirements model. Goal modelling will be one established model on which we will base our transparency model. Doing that, we aim for concepts which could be also applied on different models of an STS, e.g., those capturing dynamic aspects and business processes. We also aim to focus on the specification process of transparency as a requirement in STSs.

\section{ACKNOWLEDGEMENT}

The research is supported by an FP7 Marie Curie CIG grant (the SOCIAD project), Bournemouth University through the Fusion Investment Fund and the Graduate School Santander Grant for PGR Development.

\section{REFERENCES}

[1] G. Ropohl, "Philosophy of socio-technical systems," Techné: Research in Philosophy and Technology, vol. 4, no. 3, pp. 186-194, 1999.
[2] A. Van Lamsweerde, "Goal-oriented requirements engineering: A guided tour," in Proceedings of the Fifth IEEE International Symposium on Requirements Engineering, 2001, pp. 249-262.

[3] R. S. Aguilar-Saven, "Business process modelling: Review and framework," International Journal of production economics, vol. 90, no. 2, pp. 129-149, 2004.

[4] I. Altman, The Environment and Social Behavior: Privacy, Personal Space, Territory, and Crowding. ERIC, 1975.

[5] L. Liu, E. Yu, and J. Mylopoulos, "Security and privacy requirements analysis within a social setting," in Proceedings of the 11th IEEE International RE Conference, 2003, pp. 151-161.

[6] R. Islam, "Does more transparency go along with better governance?" Economics \& Politics, vol. 18, no. 2, pp. 121-167, 2006.

[7] F. Emery, Characteristics of sociotechnical systems. Tavistock Institute, 1959.

[8] G. McGivern and M. Fischer, "Medical regulation, spectacular transparency and the blame business," Journal of health organization and management, vol. 24, no. 6, 2010.

[9] G. McGivern and M. D. Fischer, "Reactivity and reactions to regulatory transparency in medicine, psychotherapy and counselling," Social Science \& Medicine, vol. 74, no. 3, 2012.

[10] M. D. Fischer and E. Ferlie, "Resisting hybridisation between modes of clinical risk management: Contradiction, contest, and the production of intractable conflict," Accounting, Organizations and Society, vol. 38, no. 1, pp. 30-49, 2013.

[11] J. C. S. do Prado Leite and C. Cappelli, "Software transparency," Business \& Information Systems Engineering, vol. 2, no. 3, pp. 127139, 2010.

[12] E. Yu, P. Giorgini, N. Maiden, and J. Mylopoulos, Social Modeling for Requirements Engineering: An Introduction. MIT Press, 2011.

[13] M. Turilli and L. Floridi, "The ethics of information transparency," Ethics and Information Technology, vol. 11, no. 2, pp. 105-112, 2009.

[14] C. Ball, "What is transparency?" Public Integrity, vol. 11, no. 4, pp. 293-308, 2009.

[15] D. Hess, "Social reporting and new governance regulation: The prospects of achieving corporate accountability through transparency," Business Ethics Quarterly, pp. 453-476, 2007.

[16] B. R. Rawlins, "Measuring the relationship between organizational transparency and employee trust," All Faculty Publications, 2008.

[17] J. R. Hollyer, B. P. Rosendorff, and J. R. Vreeland, "Democracy and transparency," The Journal of Politics, vol. 73, no. 04, pp. 1191-1205, 2011.

[18] J. C. S. Do Prado Leite and C. Cappelli, "Exploring i* characteristics that support software transparency." in iStar, 2008, pp. 51-54.

[19] Y.-C. Tu, C. Thomborson, and E. Tempero, "Illusions and perceptions of transparency in software engineering," in Proceedings of the 18th Asia Pacific Software Engineering Conference. IEEE, 2011, pp. 365-372.

[20] M. Serrano and J. Sampaio do Prado Leite, "Capturing transparencyrelated requirements patterns through argumentation," in Proceedings of the First International Workshop on Requirements Patterns (RePa). IEEE, 2011, pp. 32-41.

[21] C. Cappelli, J. Sampaio do Prado Leite, and A. d. P. A. Oliveira, "Exploring business process transparency concepts," in Proceedings of the 15th IEEE International RE Conference, 2007, pp. 389-390.

[22] J. Castro, M. Kolp, and J. Mylopoulos, "Towards requirements-driven information systems engineering: the tropos project," Information systems, vol. 27, no. 6, pp. 365-389, 2002.

[23] P. Bresciani, A. Perini, P. Giorgini, F. Giunchiglia, and J. Mylopoulos, "Tropos: An agent-oriented software development methodology," $\mathrm{Au}$ tonomous Agents and Multi-Agent Systems, vol. 8, no. 3, pp. 203-236, 2004.

[24] A. Stone and P. Sawyer, "Identifying tacit knowledge-based requirements," IEE Proceedings-Software, vol. 153, no. 6, pp. 211-218, 2006.

[25] R. Ali, C. Solis, I. Omoronyia, M. Salehie, and B. Nuseibeh, "Social adaptation: when software gives users a voice," in Proceedings of the 7th International Conference Evaluation of Novel Approaches to Software Engineering, June 2012, pp. 28-30.

[26] A. Finkelstein, J. Kramer, B. Nuseibeh, L. Finkelstein, and M. Goedicke, "Viewpoints: A framework for integrating multiple perspectives in system development," International Journal of Software Engineering and Knowledge Engineering, vol. 2, no. 01, pp. 31-57, 1992. 